\title{
Prediction of energy consumption using recurrent neural networks (RNN) and nonlinear autoregressive neural network with external input (NARX)
}

\author{
Wan Muhammad Zafri Wan Yahaya, Fadhlan Hafizhelmi Kamaru Zaman, Mohd Fuad Abdul Latip \\ Faculty of Electrical Engineering, Universiti Teknologi MARA, Malaysia
}

\begin{tabular}{|c|c|}
\hline Article Info & ABSTRACT \\
\hline Article history: & Recurrent Neural Networks (RNN) and Nonlinear Autoregressive Neural \\
\hline & Network with External Input (NARX) are recently applied in predicting \\
\hline Received May 18, 2019 & energy consumption. Energy consumption prediction for depth analysis of \\
\hline Revised Aug 19, 2019 & how electrical energy consumption is managed on Tower 2 Engineering \\
\hline Accepted Sep 3, 2019 & $\begin{array}{l}\text { Building is critical in order to reduce the energy usage and the operational } \\
\text { cost. Prediction of energy consumption in this building will bring great }\end{array}$ \\
\hline Keywords: & $\begin{array}{l}\text { benefits to the Faculty of Electrical Engineering UiTM Shah Alam. In this } \\
\text { work, we present the comparative study on the performance of prediction of }\end{array}$ \\
\hline Artificial intelligence & energy consumption in Tower 2 Engineering Building using RNN and \\
\hline Energy consumption & $\begin{array}{l}\text { NARX method. The model of RNN and NARX are trained using data } \\
\text { collected using smart meters installed inside the building. The results after }\end{array}$ \\
\hline Energy prediction & training and testing using RNN and NARX show that by using the recorded \\
\hline NARX & data we can accurately predict the energy consumption in the building. \\
\hline RNN & $\begin{array}{l}\text { We also show that RNN model trained with normalized data performs better } \\
\text { than NARX model. }\end{array}$ \\
\hline
\end{tabular}

Copyright $\odot 2020$ Institute of Advanced Engineering and Science. All rights reserved.

\section{Corresponding Author:}

Fadhlan Hafizhelmi Kamaru Zaman,

Faculty of Electrical Engineering, Universiti Teknologi MARA,

40450 Shah Alam, Selangor, Malaysia.

Email: fadhlan@salam.uitm.edu.my

\section{INTRODUCTION}

The population rate of humans around the world are growing rapidly every year. These phenomena will penetrate the increasing in the development and construction of building to fulfill human desire [1]. This rise also affects the electrical energy consumption among humans. Thus, concerns of energy consumption have been put into a high consideration recently in Malaysia. The energy consumption is necessary to be predicted to achieve energy conservative and reduce the cost [2-3]. On the other hands, a correct prediction of energy can be used for the future plans and provide security for the power system. The purpose of doing this study on consumption of energy is to minimize used of energy during the critical time [4-5]. Other than that, the prediction energy in buildings is very important to obtain Economical and environmental benefits It is also important to reduce Greenhouse gas emissions (GHG) such as carbon dioxide emissions [6-8] that can be considered as the main causes of global warming [9]. Several methods for predictions have been used to solve the load predicting problem. These methods can be categorized into time series and factor analysis [10]. This time series is used based on the historical load data. The factor analysis is based on several factors that can affect the energy consumption. Some of the factors are weather, numbers of human in the building and human behavior. However, the factor analysis is not easy to be implemented due to the factors related are hard to be cater to the energy consumption.

Studies on the electrical energy consumption prediction has been done by numerous methods that can be categorized into artificial intelligence (AI) methods as well as others conventional methods. Conventional methods prone to be higher in error and less accuracy compared to the AI methods. 
Conventional methods consist of three stages which includes input layer, hidden layer and output layer. This conventional method only allows one-way direction for signals to travel from input to hidden layer and then to the output. There is no feedback or loops [10]. It is a straightforward method that only involves input and output through hidden layer.

The AI methods are currently more advance than the conventional methods due to the ability to solve lots of problems that happen nowadays. There are numerous prediction techniques such as Artificial Neural Network (ANN), Bayesian Belief Network (BBN), Fuzzy Logic and Recurrent Neural Networks (RNNs) [11]. More recently, the ANN has employed a deeper architecture called Deep Layered Neural Network (DLNN) which has been used widely in many applications [12-15].

Several reviews on the methods for forecasting energy using Artificial Intelligence have been made. Recently, several researchers study about the short-term forecasting of electricity consumptions in Palestine using Artificial Neural Networks (ANN) [16]. The results when using this method shows that it is categorized as a very important tool to perform a good prediction with least mean square error as short load forecasting model. Besides, a research on prediction on rural energy consumption in Hebei Province is carried out using Gray Theory Model [17], while Suyono et al. predict the availability of solar radiation using the Extreme Learning Machine (ELM) [18]. Another research used genetic algorithm (GA) and least square support vector regression (LSSVR) for forecasting clean energy consumption [19]. Based on the research, GA-LSSVR is an effective method to reduce the degree of over-fit and also can be used as other alternatives to other clean energy consumption forecast. Particle swarm optimization is another method that is used to forecast energy demand of Turkey [20]. The result of this research shows that particle swarm optimization technique gives better forecasts than ant colony optimization technique.

\section{RESEARCH METHOD}

\subsection{Data Collection and Selection}

Data of energy consumption from tower 2 engineering building in UiTM Shah Alam were collected by using several smart meters. Data were collected on every 1 minute for about 2 months. Smart meter can be used as two ways communication between the meter and the central that is to collect and record the data in real time. Data selected must be choose based on the constraint that needs to be used for this prediction. For energy consumption prediction, 2 data have been selected as the input of the RNNs which is the voltage and current. The power data has been selected as the output of the RNNs. The input and output involve are as shown in the formula:

$$
\mathrm{P}=\mathrm{I} \times \mathrm{V}
$$

where:

$\mathrm{P}=$ power in watt $(\mathrm{W})$

$\mathrm{I}=$ current in ampere $(\mathrm{A})$

$\mathrm{V}=$ voltage in volt $(\mathrm{V})$

The input and output data must be related to get the desire results of prediction. The data used for training is Tuesday data consist of 1440 observations of data and for testing also consist 1440 observations.

\subsection{Actual and Normalized Data}

This work uses two types of data that are actual data of energy consumption and normalized data. The data that have been normalized are ranged between 0 to 1 . The formula below was used to perform the normalization.

$$
\text { norm_data }=\frac{\text { data-min }(\text { data })}{\max (\text { data })-\min (\text { data })}
$$

\subsection{Recurrent Neural Networks (RNN) and Nonlinear Autoregressive Neural Network with External Input NARX}

Recently, there has been considerable interest in the application of recurrent neural networks (RNNs) and NARX as methods for prediction. RNN and NARX has the ability to learn patterns from the past records and also to generalize and project the future load patterns for hidden data. Both RNN and NARX are networks with feedback connection. It has been proved that the proposed RNN gives a relatively accurate results for prediction [1, 21-22]. This technique is applicable to variety of problem such as speech recognition, language modeling as well as translation. In electrical field, RNN and NARX is commonly used for prediction of energy consumption, energy efficiencies and load demand. Recent study the RNN and 
NARX method is developed in order to forecast the energy consumption in [23-25]. This model can predict energy consumption and will help in developing highly applicable energy policies in Iran. Another research in 2014 by Chen Fang using Elman Recurrent Neural Network (ERNN) applied on specific humidity forecasting. This model of ERNN builds to predict the specific humidity from three weather stations. The results on this research shows that this ERNN model has great potential than Multilayer perceptron (MLP) during winter season when the data noise produced is very small [26]. This result shows that ERNN can be an alternative method to predict specific humidity.

Based on Figure 1, this work uses 2 inputs, 1 output and 10 and 20 hidden layers for RNN and NARX respectively. NARX network has and external input with delay of 20. Both the RNN and NARX are trained using the Levenberg-Marquardt (trainlm) for as training function. The construction of network models in MATLAB is shown below.
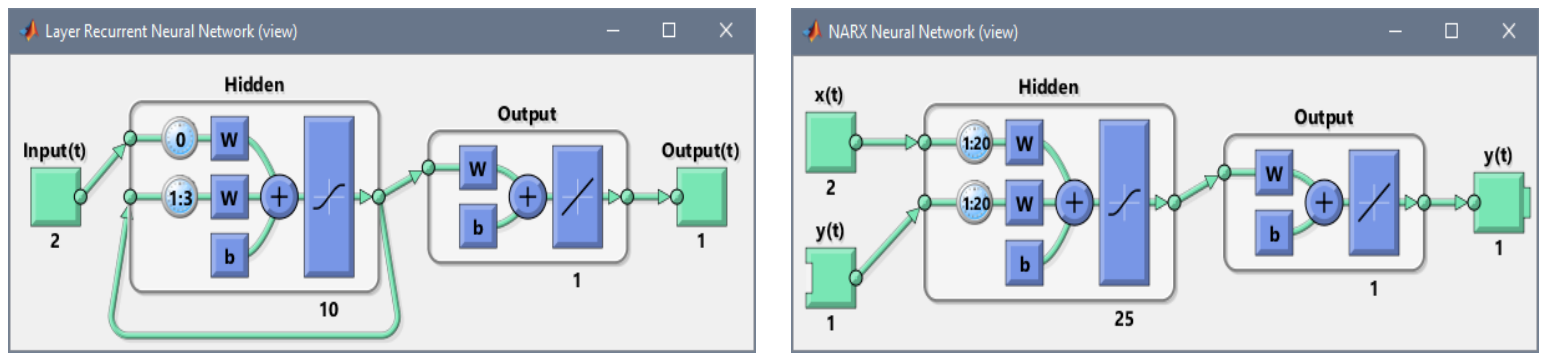

Figure 1. Construction of RNN (top) and NARX (bottom) models used in this work

RNN: layrecnet(layerDelays,hiddenSizes,trainFcn)

NARX: narxnet(inputDelays,feedbackDelays,hiddenSizes,trainFcn)

where:

layerDelays $=$ row vector of increasing 0 or positive delays $($ default $1: 2)$

hiddenSizes $=$ row vector of one or more hidden layer sizes $($ default $=10)$

trainFcn $=$ training function (default $=$ 'trainlm')

The procedure of training and testing are explained in steps below:

a. Load the energy consumption data collected on Tuesday $25^{\text {th }}$ July 2017 that consist the value voltage and current as input, while the value of power as output

b. The data were trained using layer recurrent neural network (layrecnet) and NARX (narxnet). The delay used is 3 for RNN and 20 for NARX.

c. After training, the predicted data was compared with the actual data.

d. The training result was analyzed using histogram for the error and means square error (MSE) value was calculated.

e. Load the energy consumption data collected on Thursday $27^{\text {th }}$ July 2017 for testing.

f. The test result has also been analyzed using histogram for the error and means square error (MSE) value was calculated.

\subsection{Process Flow}

The flow chart shown in Figure 2 is the overall process of RNN and NARX method for prediction of energy consumption in Tower 2 Engineering Building in UiTM Shah Alam. The overall process consists of the data collection and selection based on the constraint, training process, testing process as well as comparison and bench-marking of the result. 


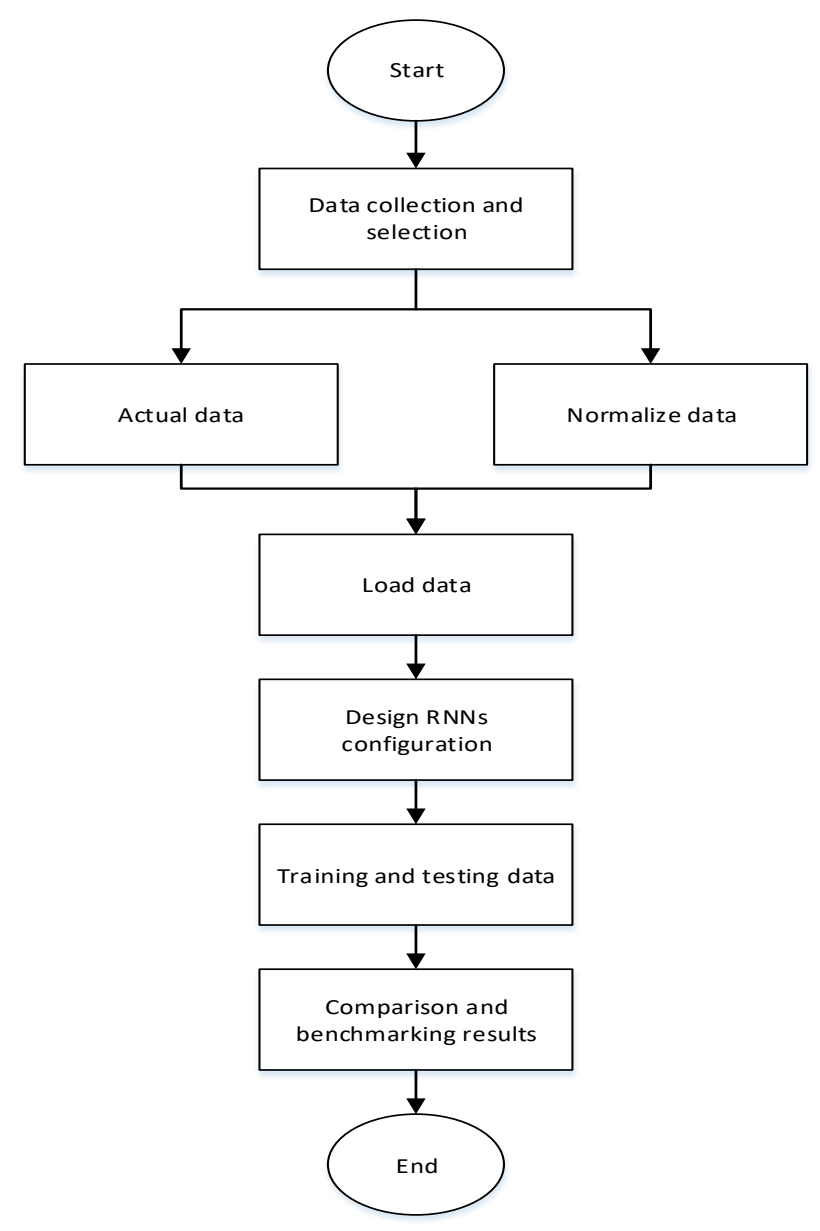

Figure 2. Flow chart for RNN and NARX implementation

\section{RESULTS AND ANALYSIS}

In this work, a trial and error techniques has been used to get the suitable number of hidden layer for both RNN and NARX networks. For RNN implementation, we simply start with five hidden layers and increases by five layers up until twenty layers. For NARX, we start with five hidden layers, and increase the layers gradually by 5 layers up to 25 layers. Then, the training performance for RNN and NARX implementation are collected based on the Means Square Error (MSE) values. Based on the results tabulated in Table 1 and Table 2, MSE for training generally decreases when the number of hidden layer increases. Thus, it can be concluded that for training, the number of hidden layers is inversely proportional to the MSE. The lowest MSE recorded for RNN and NARX is at 10 hidden layers and 20 hidden layers respectively, which yield MSE of 0.5689 and 37.3488 respectively. For RNN the highest MSE recorded is at 5 hidden layers which is 1.4065 , indicating that too small hidden layers are unable to learn the underlying patterns. However, selection of the number of hidden layers should be determined carefully, since when the number of hidden layers are more than necessary, the network will overfit and fail to learn the inherent pattern. Instead it will generalize too well on the training data and fail on testing data. Based on the Table 1 and Table 2, the optimal number of hidden layers used in this work for RNN and NARX is 10 and 20 respectively.

\subsection{Test Results Using Actual data}

The implementation of RNN and NARX models for prediction contains two test parts, where we report the results using an actual data and normalized data. For the first part, the result for test data was obtained through the graph in Figure 3, Figure 4 and Figure 5. The actual output is plotted versus the predicted output on the same graph. We can observe that RNN model gave better predicted values when compared to NARX. RNN test performance reported as MSE value is 55.834, where NARX performance is 58.145. Based on Figure 4, comparison between the actual and predicted values shows some errors are apparent. For an ideal model, the predicted plot would overlay the actual plot completely. The errors for the 
test result between actual output and predicted output were shown for both RNN and NARX in histogram graph in Figure 5. The histogram graph can be used to record the frequent numbers of errors in the testing result. Based on Figure 4 the error for RNN test result shows that it lies between -6 to 4 and the most distribution is at 0 . While for NARX, the error has a wider spread between -30 to 30 , indicative that RNN performs significantly better than NARX for actual data usage.

Table 1. Selection of Hidden Layer based on MSE Performance for RNN

\begin{tabular}{ccc}
\hline Number of hidden layers & \multicolumn{2}{c}{ MSE } \\
& RNN & RNN (Normalized) \\
\hline 5 & 1.4065 & 0.4851 \\
10 & 0.5689 & 0.5340 \\
15 & 0.7990 & 0.7219 \\
20 & 0.8696 & 0.8836 \\
\hline
\end{tabular}

Table 2. Selection of Hidden Layer based on MSE Performance for NARX

\begin{tabular}{ccc}
\hline Number of hidden layers & \multicolumn{2}{c}{ MSE } \\
& NARX & NARX (Normalized) \\
\hline 5 & 41.3837 & 37.4890 \\
10 & 38.1679 & 35.6996 \\
15 & 43.7693 & 39.4053 \\
$\mathbf{2 0}$ & 37.3488 & 38.3441 \\
25 & 40.3913 & 31.5778 \\
\hline
\end{tabular}

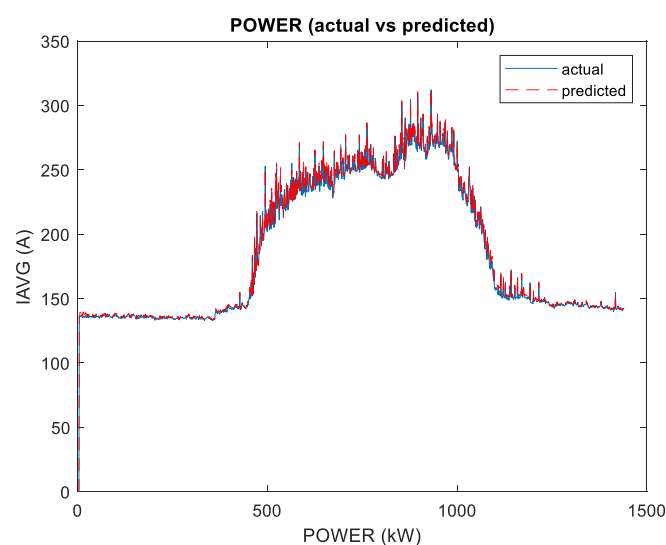

RNN

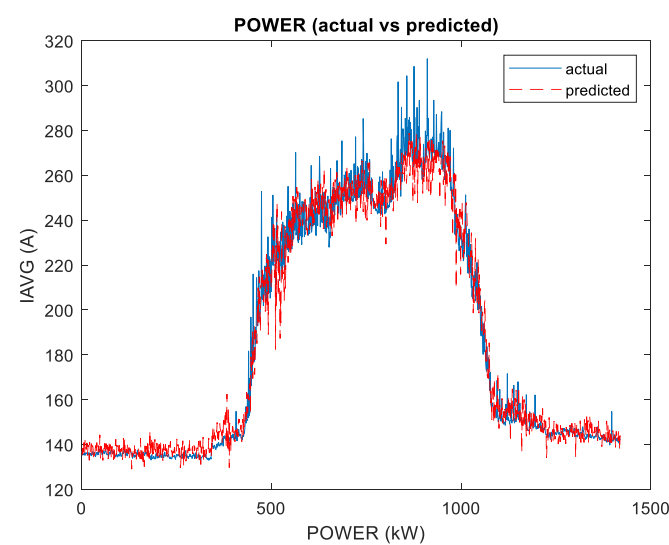

NARX

Figure 3. Test result showing the actual and predicted data of power $(\mathrm{kWh})$

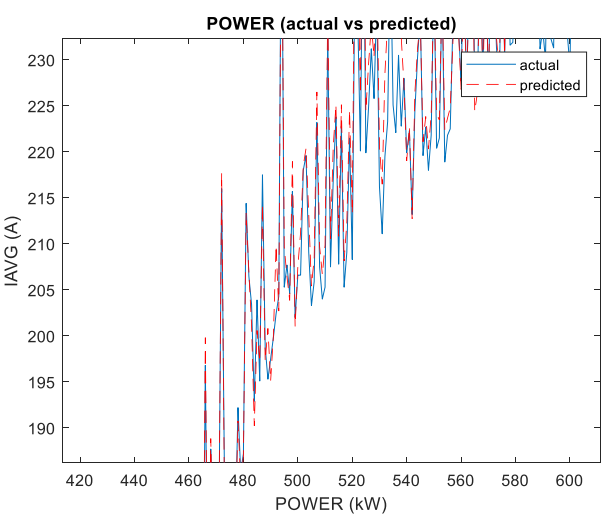

RNN

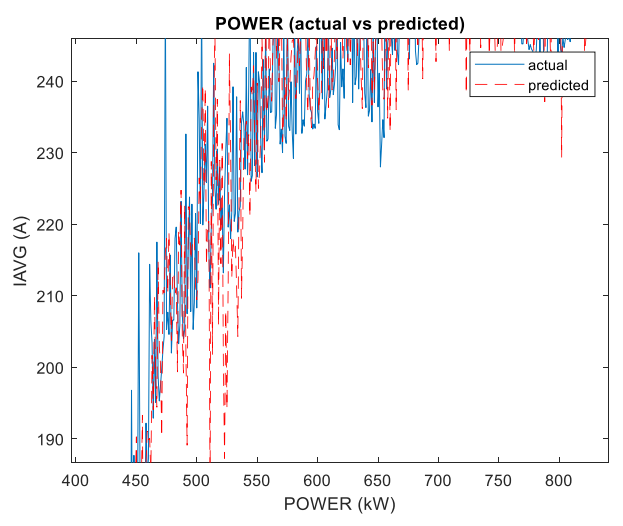

NARX

Figure 4. Magnified view of test result showing actual and predicted data of power $(\mathrm{kWh})$

Prediction of energy consumption using recurrent neural networks... (Wan Muhammad Zafri Wan Yahaya) 

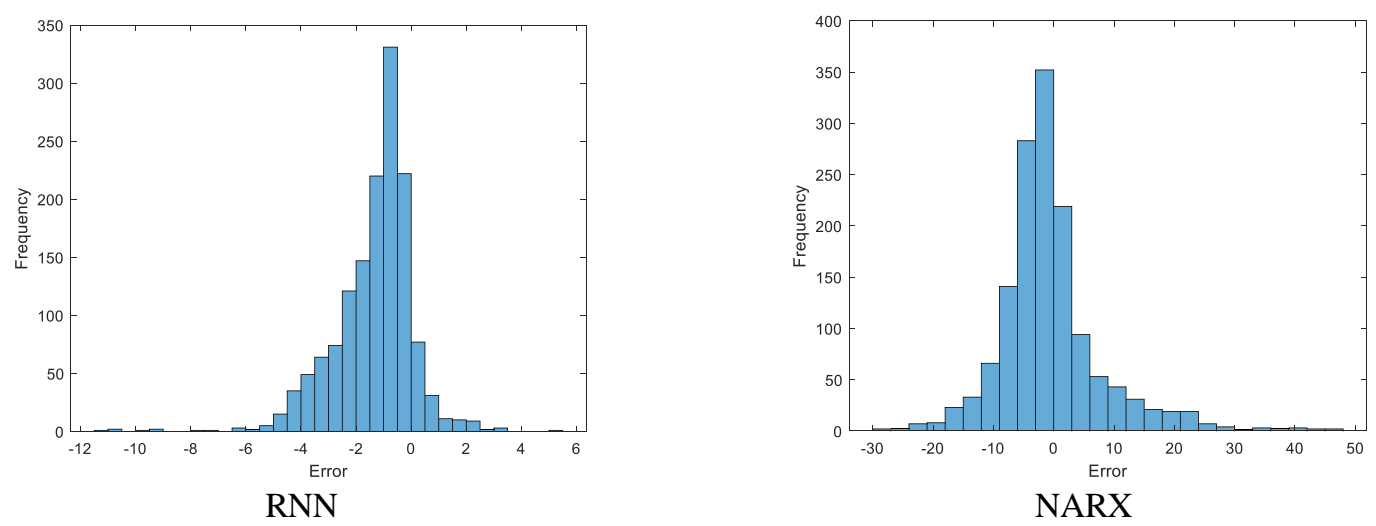

Figure 5. The errors between actual and predict data illustrated as error histogram

\subsection{Test result using normalized data.}

For the second part, the result of test data was obtained from normalized data. The results are shown in Figure 6, Figure 7 and Figure 8. Likewise, the actual output is plotted versus the predicted output. According to Figure 7, RNN model yields better prediction than NARX model. The outlying normalized and predicted data is better in RNN case, while for NARX, the prediction contains large errors. In fact, the performance of this RNN in term of MSE is 42.215, while for NARX the performance is 60.267. For RNN model, the shape of predicted graph lies below the actual graph. The errors for the testing result between actual output and predicted output were shown in histogram graph in Figure 8. Based on Figure 7 the error for RNN in this test result shows that it lies between -10 to 10 and the most distribution is at 0 . Larger error observed in NARX model where the error is spread between -20 to 40 .
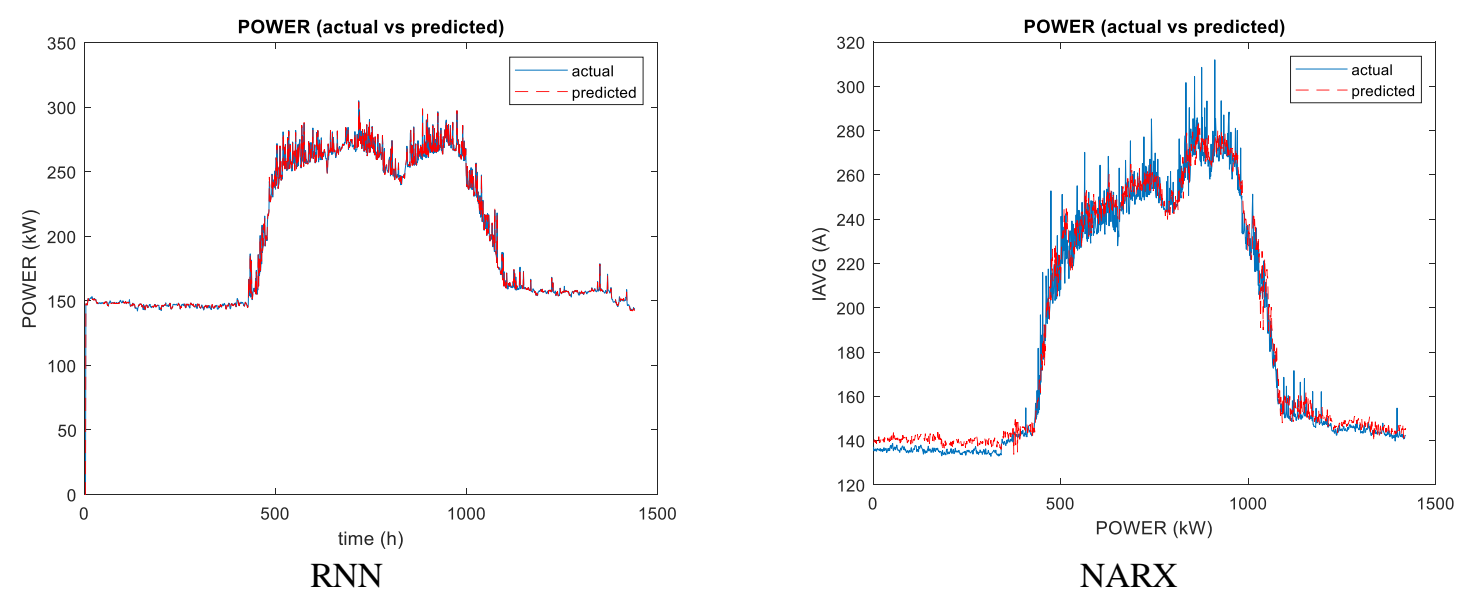

Figure 6. Test result showing the actual and predicted data of power (kWh)

Further analysis on the results compares the MSE performance for both RNN and NARX model in actual and normalized data usage cases. This is tabulated in Table 3. Based on Table 3, the best overall performance is achieved by RNN model which uses normalized data, where it delivers lowest error of 42.215. The worst model is NARX model using normalized data, which yields MSE of 60.267. Figure 9 shows larger magnification of RNN model prediction, to highlight its accuracy.

Table 3. MSE performance for RNN and NARX on test data

\begin{tabular}{ccc}
\hline Data & RNN & NARX \\
\hline Actual & 55.834 & 58.145 \\
Normalized & 42.215 & 60.267 \\
\hline
\end{tabular}




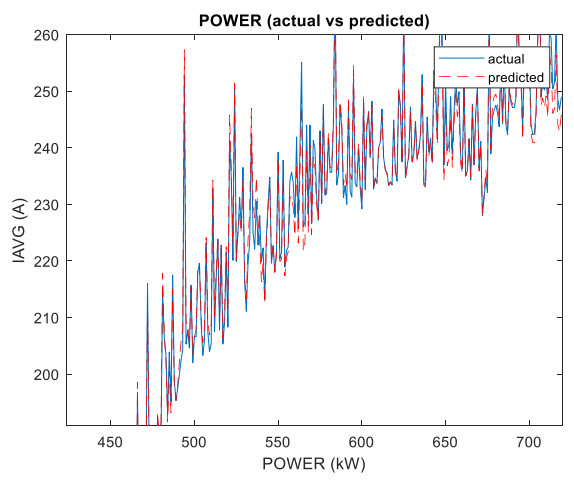

RNN

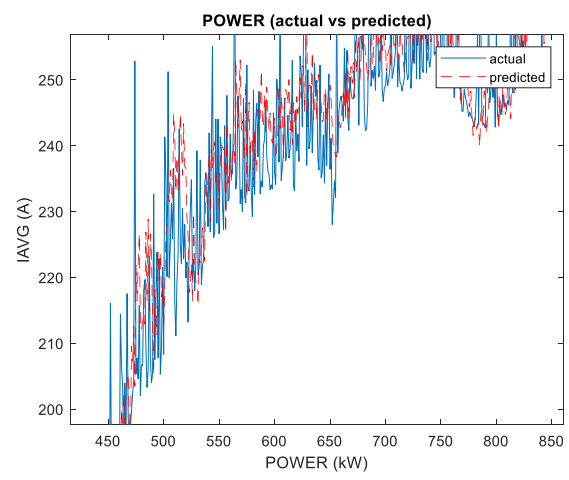

NARX

Figure 7. Magnified view of test result showing actual and predicted data of power (kWh)

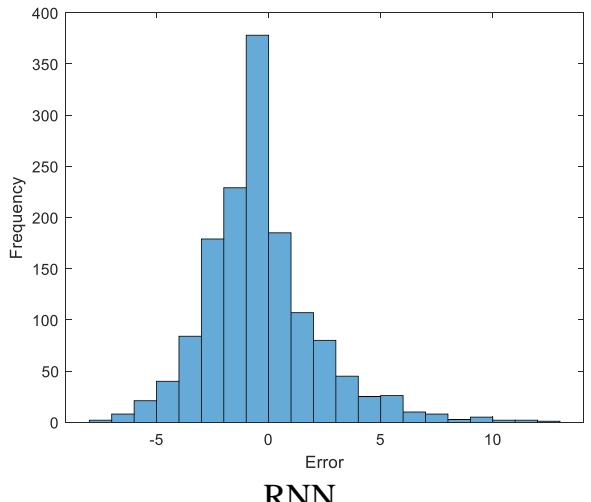

RNN

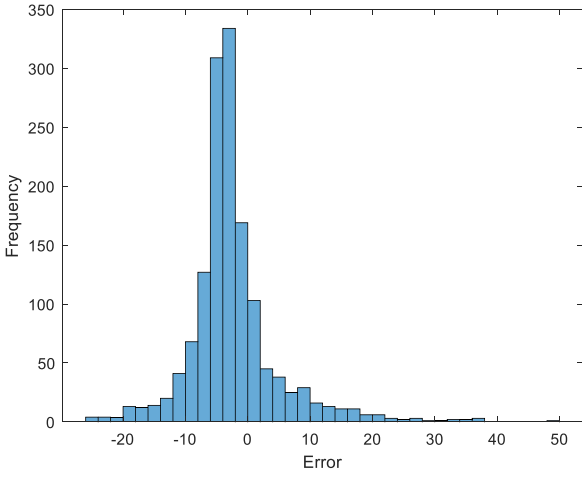

NARX

Figure 8. The errors between actual and predict data illustrated as error histogram

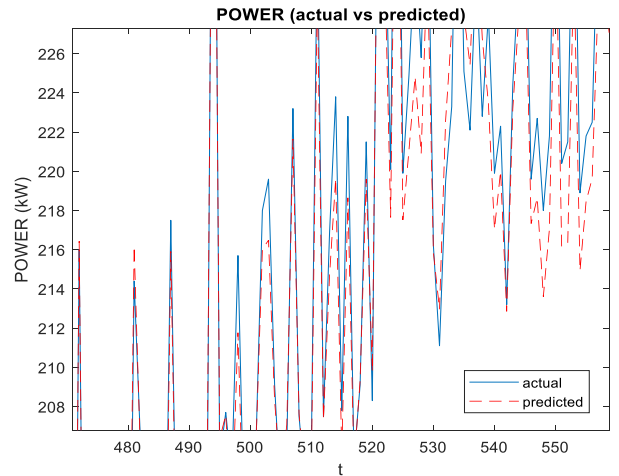

Figure 9. Larger magnification of RNN model result, showing relatively accurate prediction

\section{CONCLUSION}

In In this paper, two models namely RNN model and NARX model was trained for prediction of energy consumption in Tower 2 Engineering Building at UiTM Shah Alam. Two historical data sets have been used and analysed on this model for data of energy consumption which are data collected on Tuesday 25th July 2017 and on Thursday 27th July 2017. The prediction process involves three stages which is the selection of data based on constraint, training, as well as testing. Then, to select the best hidden layer, trial and error technique is used. The best number of hidden layers is 10 for RNN and 20 for NARX, which is according to experiments conducted. The results obtained after training and testing for both actual and normalized data, shows that RNN model with normalized data delivers the best performance with MSE of

Prediction of energy consumption using recurrent neural networks... (Wan Muhammad Zafri Wan Yahaya) 
42.215. Further enhancement to the work here can be achieved by using larger data such as data of a month or of a year energy consumption of this building. Other than that, continuation of this study on a new method for prediction that may give more accurate results for energy predictions.

\section{ACKNOWLEDGEMENTS}

This research is funded by Faculty of Electrical Engineering, UiTM Shah Alam, Selangor, Malaysia. The authors also gratefully acknowledge Faculty of Electrical Engineering UiTM Shah Alam for all the support given during this research.

\section{REFERENCES}

[1] Avami, A., \& Boroushaki, M. (2011). "Energy consumption forecasting of Iran using recurrent neural networks". Energy Sources, Part B: Economics, Planning and Policy, 6(4), 339-347. https://doi.org/10.1080/15567240802706734

[2] Platon, R., Dehkordi, V. R., \& Martel, J. (2015). "Hourly prediction of a building's electricity consumption using case-based reasoning, artificial neural networks and principal component analysis". Energy and Buildings, 92, 10-18. https://doi.org/10.1016/j.enbuild.2015.01.047

[3] F. Shrouf, J. Ordieres-meré, A. García-sánchez, and M. Ortega-mier, “Optimizing the production scheduling of a single machine to minimize total energy consumption costs," J. Clean. Prod., vol. 67, pp. 197-207, 2014.

[4] L. Yang, H. Yan, and J. C. Lam, "Thermal comfort and building energy consumption implications-A review," Appl. Energy, vol. 115, pp. 164-173, 2014.

[5] S. Bilgen, "Structure and environmental impact of global energy consumption," Renew. Sustain. Energy Rev., vol. 38, pp. 890-902, 2014.

[6] A. Marvuglia and A. Messineo, "Using recurrent artificial neural networks to forecast household electricity consumption," Energy Procedia, vol. 14, pp. 45-55, 2012.

[7] A. Greiner, L. Gruene, and W. Semmler, "Schwartz Center For Economic Policy Analysis The New School Economic Growth and the Transition from Non-Renewable to Renewable Energy," no. August, 2012

[8] P. Nejat, F. Jomehzadeh, M. Mahdi, and M. Gohari, “A global review of energy consumption, CO 2 emissions and policy in the residential sector ( with an overview of the top ten CO 2 emitting countries )," Renew. Sustain. Energy Rev., vol. 43, pp. 843-862, 2015.

[9] Soytas, U., Sari, R., \& Ewing, B. T. (2007). "Energy consumption, income, and carbon emissions in the United States". Ecological Economics, 62(3-4), 482-489. https://doi.org/10.1016/j.ecolecon.2006.07.009

[10] Rashid, T., \& Huang, B. (2006). "Auto-regressive recurrent neural network approach for electricity load forecasting". International Journal of Electrical and Computer Engineering (IJEECS), 3(1), 36-44. Retrieved from http://citeseerx.ist.psu.edu/viewdoc/download?doi=10.1.1.136.7164\&rep=rep1\&type=pdf

[11] Khan, M. J., Shamail, S., Awais, M. M., \& Hussain, T. (2006). "Comparative Study of Various Artificial Intelligence".

[12] R. Girshick, J. Donahue, T. Darrell, and J. Malik, "Region-Based Convolutional Networks for Accurate Object Detection and Segmentation," IEEE Transactions on Pattern Analysis and Machine Intelligence, vol. 38, no. 1, pp. 142-158, 2016.

[13] J. Redmon and A. Farhadi, "YOLOv3: An Incremental Improvement," ArXiv e-prints, vol. 1804.02767, 2018.

[14] Y. LeCun, Y. Bengio, and G. Hinton, "Deep learning," Nature, Insight vol. 521, no. 7553, pp. 436-444, 05/28/print 2015.

[15] L. Deng and D. Yu, "Deep Learning: Methods and Applications," Foundations and Trends in Signal Processing, vol. 7, pp. 3-4, 2014.

[16] Riflet, G. (2017). "F Orecasting O Ffshore". Energy, 8(2), 11-21.

[17] Cui He-rui*, Zhang Peng-yu, "Prediction of Rural Energy Consumption Based on the Gray Theory in Hebei Province ", TELKOMNIKA Indonesian Journal of Electrical Engineering Vol.12, No.3, March 2014, pp. 2314-2319

[18] Hadi Suyono, Hari Santoso, Rini Nur Hasanah, Unggul Wibawa, Ismail Musirin, "Prediction of Solar Radiation Intensity using Extreme Learning Machine", Indonesian Journal of Electrical Engineering and Computer Science (IJEECS), Vol. 12, No. 2, November 2018, pp. 691-698.

[19] Wang, Z., Shi, J., Dai, W., Wu, J., \& Tang, L. (2013). "Clean Energy Consumption Forecast Based on GA-LSSVR Hybrid Learning Paradigm". 2013 Sixth International Conference on Business Intelligence and Financial Engineering, (1), 139-142. https://doi.org/10.1109/BIFE.2013.31

[20] Paksoy, T., \& Weber, G. (2012). "Particle Swarm Optimization Approach for Estimation of Energy Demand of Turkey". Global Journal of Technology \& Optimization, 3(June), 1-9.

[21] K. Benmouiza and A. Cheknane, "Forecasting hourly global solar radiation using hybrid k -means and nonlinear autoregressive neural network models," Energy Convers. Manag., vol. 75, pp. 561-569, 2013.

[22] H. E. Consumption, "Energy Procedia Using Recurrent Artificial Neural Networks to Forecast Household Electricity Consumption," 2012.

[23] Kermanshahi, B. (1998). "Recurrent neural network for forecasting next 10 years loads of nine Japanese utilities". Neurocomputing, 23(1-3), 125-133. https://doi.org/10.1016/S0925-2312(98)00073-3 
[24] G. Capizzi, C. Napoli, and F. Bonanno, "Innovative Second-Generation Wavelets Construction With Recurrent Neural Networks for Solar Radiation Forecasting," vol. 23, no. 11, pp. 1805-1815, 2012.

[25] M. Ibrahim, S. Jemei, G. Wimmer, and D. Hissel, "Nonlinear autoregressive neural network in an energy management strategy for battery / ultra-capacitor hybrid electrical vehicles," Electr. Power Syst. Res., vol. 136, pp. 262-269, 2016.

[26] Fang, C., Wang, X., Murphey, Y. L., Weber, D., \& Macneille, P. (2014). "Specific Humidity Forecasting using Recurrent Neural Network."

\section{BIOGRAPHIES OF AUTHORS}
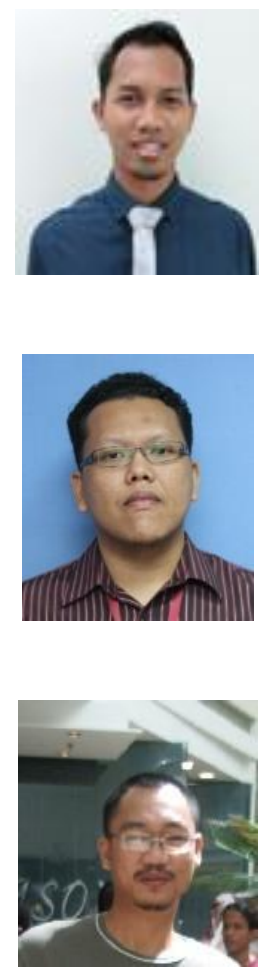

Wan Muhammad Zafri Wan Yahaya received Diploma in Electrical Engineering from Universiti Teknologi Mara (UiTM) Pulau Pinang and B.Sc (Hons) Electrical Engineering from Universiti Teknologi Mara (UiTM) Shah Alam in 2012 and 2017. He is currently working as Process Engineer at Southern Cable Sdn. Bhd. His research interest in energy consumption, prediction and artificial intelligence.

Fadhlan Hafizhelmi Kamaru Zaman received the B.Sc (Hons.) and P.hD. degrees from International Islamic University Malaysia in 2008 and 2015, respectively. He is currently a Senior Lecturer at Department of Computer Engineering, University of Technology MARA, Malaysia. His research interests are in surveillance system, pattern recognition, signal and image processing, artificial intelligence and computer vision. Fadhlan is also a member of IEEE, Malaysian Board of Technologist (MBOT), and a Chartered Engineer from the Institution of Engineering and Technology, UK.

Mohd Fuad Abdul Latip received his B.Eng. (Electrical Engineering) and M.Eng (Electrical Energy and Power System) degrees from the University of Malaya, Malaysia in 2000 and 2006, respectively. He is a senior lecturer at the Faculty of Electrical Enginering, Universiti Teknologi MARA, Shah Alam, Malaysia. He was an engineer at Matsushita TV \& Network and Celcom (M) Bhd in 2001 and 2002, respectively. His main interest research are Energy efficiency, saving and monitoring, application of computer and IOT in power system and Artificial intelligence for electrical energy optimization. He is a member of IEEE, IAENG and MBOT. 